\title{
La prueba del Q-TIP en la valoración de la mujer con incontinencia urinaria de esfuerzo
}

\author{
Antonio Lomanto Morán*; Mario Orlando Parra Pineda**; Jacinto Sánchez Angarita***
}

\begin{abstract}
RESUMEN: Se investigó la movilidad de la unión uretro-vesical mediante la prueba del Q-TIP en mujeres con incontinencia urinaria de esfuerzo $(n=60)$, en mujeres sin incontinencia urinaria $(n=41)$ y en mujeres con otros tipos de incontinencia urinaria 0 ya tratadas $(n=33)$. La prueba del Q-TIP fue positiva en el $58 \%$ de las mujeres, sin encontrarse diferencias significativas entre las mujeres con incontinencia urinaria de esfuerzo y sin incontinencia $(p=0.104)$. La prueba del Q-TIP estuvo estrechamente relacionada con el grado de uretrocistocele de las pacientes (grado 0: $10 \%$, grado I: $53 \%$, grado II: 60\% y grado III: $90 \%$ ). Se concluye que la prueba del Q-TIP guarda estrecha relación con el grado de uretrocistocele, sin ninguna correlación con un diagnóstico urológico específico.
\end{abstract}

PALABRAS CLAVES: Incontinencia urinaria de esfuerzo, Prueba del Q-TIP, Uretrocistocele.

SUMMARY: The mobility of the urethra was studied using the Q-TIP test in women with genuine stress urinary incontinence (n=60), continente women $(n=41)$ and women with other types of urinary incontinence or women already treated $(n=33)$. The $Q-T I P$ test was positive in $58 \%$ of the women, no significant differences between women with stress urinary incontinence and continent women were found. ( $p=$ 0.104). The Q-TIP test was closely related with the urethrocystocele degree of the patients (degree $0: 10 \%$, degree I: $53 \%$, degree II: $60 \%$ and degree III: $90 \%$ ). In conclusion, the Q-TIP test has a close relationship with the urethrocystocele degree, but it doesn't have any correlation with a specific urologic diagnosis.

KEY WORDS: Stress urinary incontinence, the Q-TIP test, Urethrocystocele.

\section{Introducción}

La prueba del Q-TIP fue descrita por Crystle y colaboradores en 1971 como un método alterno, sencillo y económico, para la valoración de la movilidad y de la unión uretrovesical, tradicionalmente evaluada por el uretrocistograma de cadena y clasificada según los criterios de Green (Tipos 1 y 2), ya abandonados en la práctica clínica $(1-2,12)$.

La utilidad clínica de la prueba en la valoración prequirúrgica de la paciente con incontinencia urinaria de esfuerzo ha sido la de cuantificar objetivamente el grado de movilidad de la unión uretrovesical; establecer la necesidad de exámenes urodinámicos adicionales; ayudar a determinar el tipo de cirugía correctora y servir como factor pronóstico del éxito del tratamiento quirúrgico (1, 6-7). En la valoración postquirúrgica adicionalmente la prueba cuantifica el grado de corrección en el desplazamiento de la unión uretrovesical alcanzado en el acto quirúrgico.

Sin embargo, la utilidad de esta prueba en el estudio de la paciente con incontinencia urinaria de esfuerzo ha sido

\footnotetext{
* Profesor Asociado. Departamento de Ginecología y Obstetricia. Facultad de Medicina. Universidad Nacional de Colombia. Clínica de Incontinencia Urinaria. Instituto Materno Infantil.

** Docente Ocasional. Departamento de Ginecología y Obstetricia. Facultad de Medicina. Universidad Nacional de Colombia.

*** Profesor Asistente. Departamento de Ginecología y Obstetricia. Facultad de Medicina. Universidad Nacional de Colombia. Clínica de Incontinencia Urinaria. Instituto Materno Infantil.
}

muy cuestionada. Caputo y colaboradores al compararla con el estudio ultrasonográfico en la evaluación objetiva de la movilidad de la unión uretrovesical, encontraron que la prueba del Q-TIP es inexacta (sensibilidad 25\%, especificidad $78 \%$ ). Walters y colaboradores al valorar la prueba en mujeres con incontinencia urinaria encontraron que este método está relacionado con defectos en el soporte de la pared vaginal anterior, más no con un diagnóstico urológico específico (3-4, 10-11).

El objetivo de este trabajo fue determinar la utilidad de la prueba del Q-TIP en la valoración de las pacientes con incontinencia urinaria de esfuerzo y establecer su relación con el grado de uretrocistocele de la mujer.

\section{Material y métodos}

El trabajo corresponde a un estudio de tipo descriptivo y prospectivo, en el que se valoraron 134 pacientes que asistieron a la consulta de Ginecología o a la Clínica de Incontinencia Urinaria del Departamento de Ginecología y Obstetricia de la Universidad Nacional en el Instituto Materno Infantil, en el período de tiempo comprendido entre el 1o. de julio de 1994 y 31 de enero de 1995.

A las pacientes se les realizó una anamnesis y examen físico completos, incluyendo la prueba del Q-TIP y estudios adicionales como urocultivo, cistoscopia y urodinamia de acuerdo con las normas de manejo en laClínica de Incontinencia Urinaria, a fin de descartar o confirmar en forma definitiva el diagnóstico de incontinencia urinaria de esfuerzo. 
La prueba del Q-TIP se realizó como se describe a continuación:

- Colocación de la paciente en posición ginecológica.

- Valoración del grado de uretrocistocele (Grado 0, no hay prolapso de la mitad inferior de la pared vaginal anterior; Grado I, hay prolapso hasta el tercio inferior de la vagina; Grado II, hay prolapso que llega hasta el introito vaginal; Grado III, hay prolapso que sobrepasa el introito vaginal) y reducción del mismo.

- Asepsia del meato uretral con solución de yodo polividona.

- Lubricación de la punta del aplicador con lidocaína jalea al $2 \%$.

- Introducción suave del aplicador hasta la vejiga y posterior retiro del mismo hasta encontrar resistencia, a fin de localizar el extremo del aplicador a nivel de la Medición del ángulo que forma el aplicador con respecto a la horizontal, mientras la paciente está en reposo.

- Medición del ángulo máximo que forma el aplicador con respecto a la horizontal, mientras la paciente realiza la maniobra de valsalva en forma sostenida.

El grado de movilidad de la unión uretrovesical se obtiene de la diferencia entre el ángulo máximo conseguido durante la maniobra de Valsalva y el ángulo en reposo. En nuestra clínica de incontinencia urinaria este ángulo se obtiene directamente a través de un goniómetro modificado.

Se considera que la prueba del Q-TIP es positiva cuando el ángulo resultante es mayor o igual a $30^{\circ}$ (1$2,9,12$ ).

A los datos obtenidos se les aplicó la prueba de significancia estadística Chi cuadrado, y se consideraron significativas las diferencias con $\mathrm{p} \leq 0.05$.

\section{Resultados}

Se realizaron 134 pruebas del Q-TIP, distribuidas así: 60 $(45 \%)$ en pacientes con incontinencia urinaria de esfuerzo pura (I.U.E.); 41 (32\%) en pacientes ginecológicas sin incontinencia urinaria (NO I:U.) y 33 (23\%) en pacientes con incontinencia urinaria de otra etiología o ya tratadas quirúrgicamente (OTRAS) (Figura 1).

La edad promedio de estas pacientes fue de 49 años (rango: $26-77$ años) y la paridad de 4.5 (rango: $0-15$ partos), sin encontrar diferencias estadísticamente significativas de edad o paridad entre los grupos (P: 0.95) (Tabla 1).

Tabla 1

PRUEBA DEL Q-TIP

\begin{tabular}{|lcl|}
\hline Pacientes & Edad* & Paridad* \\
\hline I.U.E. & $49(27-70)$ & $4.7(0-15)$ \\
NO I.U. & $48(26-70)$ & $4.2(0-11)$ \\
OTRAS & $51(27-77)$ & $4.7(0-12)$ \\
PROMEDIO & $49(26-77)$ & $4.5(0-15)$ \\
\hline
\end{tabular}

* Promedio y rango

$\mathrm{P}=0.95$

En el examen físico la frecuencia de uretrocistocele en las pacientes con incontinencia urinaria de esfuerzo, fue del $92 \%$ y en las pacientes sin incontinencia urinaria fue del $85 \%$, siendo de los más frecuentes el grado II y I, en ambos grupos. No se encontraron diferencias estadís-
Figura 1

PRUEBA DEL Q-TIP. DIAGNOSTICO CLINICO

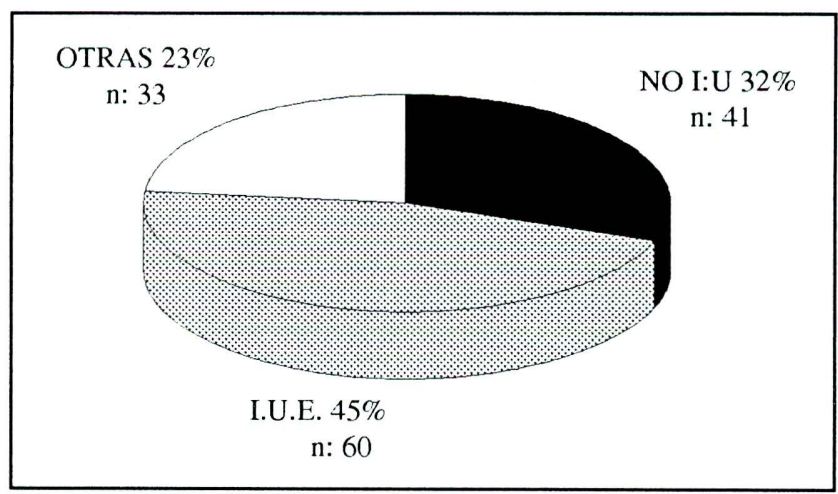

ticamente significativas en la frecuencia y los grados de uretrocistocele entre estos dos grupos de pacientes ( $\mathrm{p}$ : 0.53 ) (Figura 2).

La prueba del Q-TIP fue positiva en el $65 \%$ de las pacientes, con incontinencia urinaria de esfuerzo y en el 49\% de las pacientes sin incontinencia urinaria, sin encontrarse diferencias estadísticamente significativas en la positividad de la prueba entre estos dos grupos de pacientes (p: 0.104) (Figura 3).

La prueba del Q-TIP como método de diagnóstico de incontinencia urinaria de esfuerzo tuvo una sensibilidad del $65 \%$, una especificidad del $51 \%$, un valor predictivo positivo del $66 \%$ y un valor predictivo negativo del $50 \%$.

La prueba del Q-TIP estuvo estrechamente relacionada con el grado de uretrocistocele de la paciente es así como fue positiva en el $10 \%$ de las pacientes sin uretrocistocele; en el $53 \%$ de las pacientes con uretrocistocele grado I; en el $60 \%$ de las pacientes con uretrocistocele grado II y en el $90 \%$ de las pacientes con uretrocistocele grado III. Sin encontrarse diferencias estadísticamente significativas en la positividad de la prueba con relación a la frecuencia y grado del uretrocistocele entre las pacientes con o sin incontinencia urinaria de esfuerzo (p 0.61) (Figuras 4 y 5).

\section{Figura 2}

PRESENCIA Y GRADO DEL URETROCISTOCELE EN PACIENTES CON INCONTINENCIA URINARIA DE ESFUERZO Y SIN INCONTINENCIA URINARIA

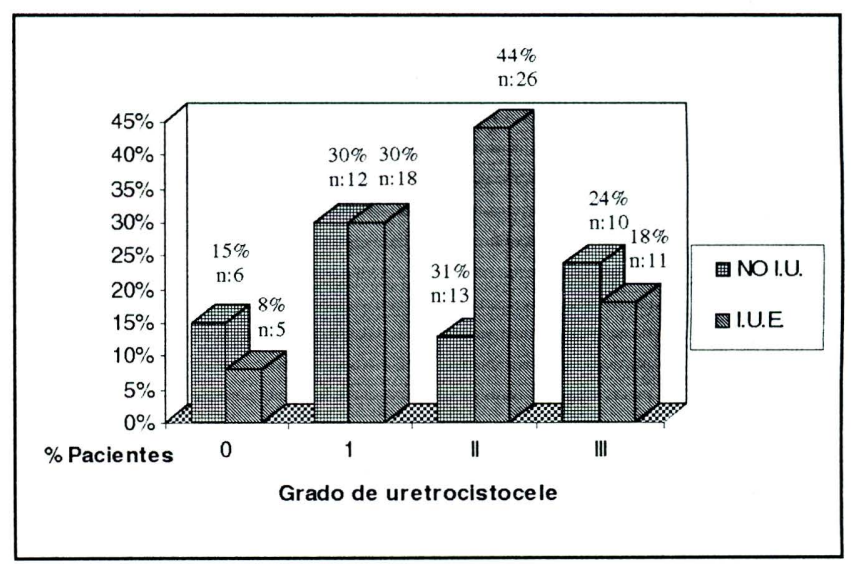


Figura 3

LA PRUEBA DEL Q-TIP EN PACIENTES CON INCONTINENCIA URINARIA DE ESFUERZO Y SIN INCONTINENCIA URINARIA

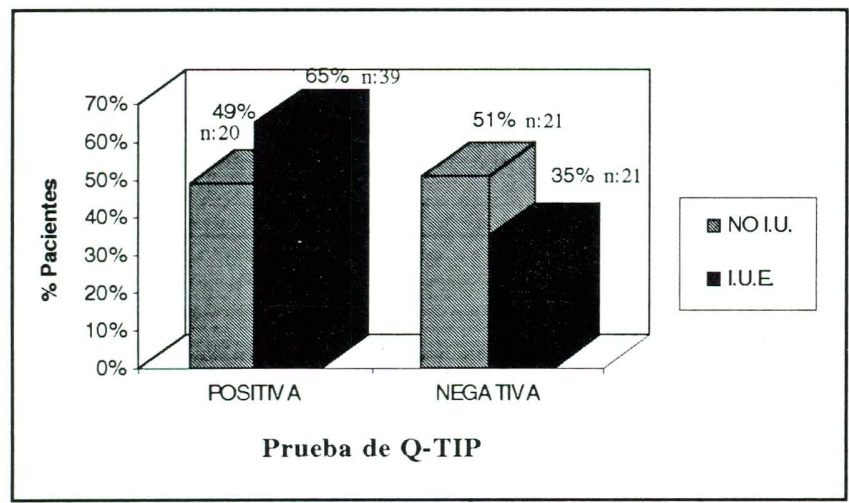

Figura 5

RELACION ENTRE LA PRUEBA DEL Q-TIP POSITIVA Y EL GRADO DE URETROCISTOCELE EN PACIENTES CON INCONTINENCIA URINARIA DE ESFUERZO Y SIN INCONTINENCIA URINARIA

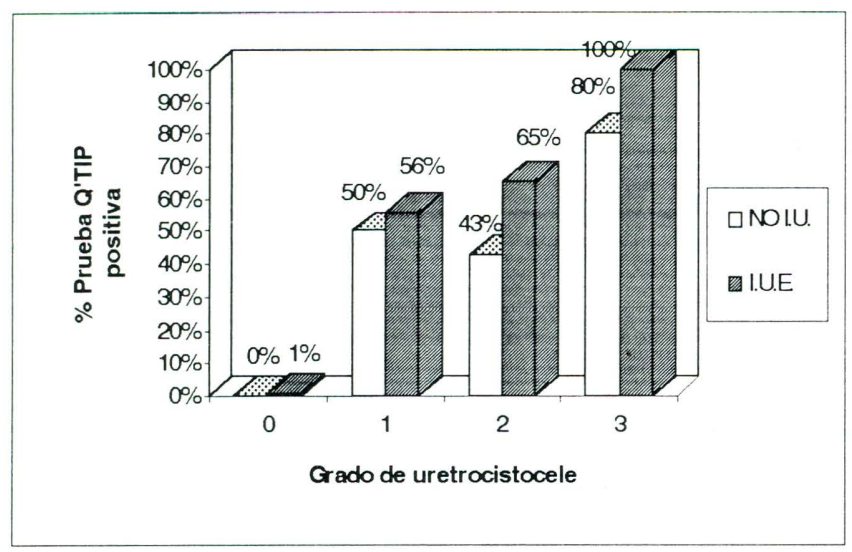

\section{Análisis}

El grupo de pacientes estudiadas corresponden a mujeres premenopáusicas, con alta paridad y una gran incidencia de prolapso de la pared vaginal anterior, características epidemiológicas similares a las encontradas por otros investigadores.
Figura 4

RELACION ENTRE LA PRUEBA DEL Q-TIP POSITIVA Y EL GRADO DE URETROCISTOCELE

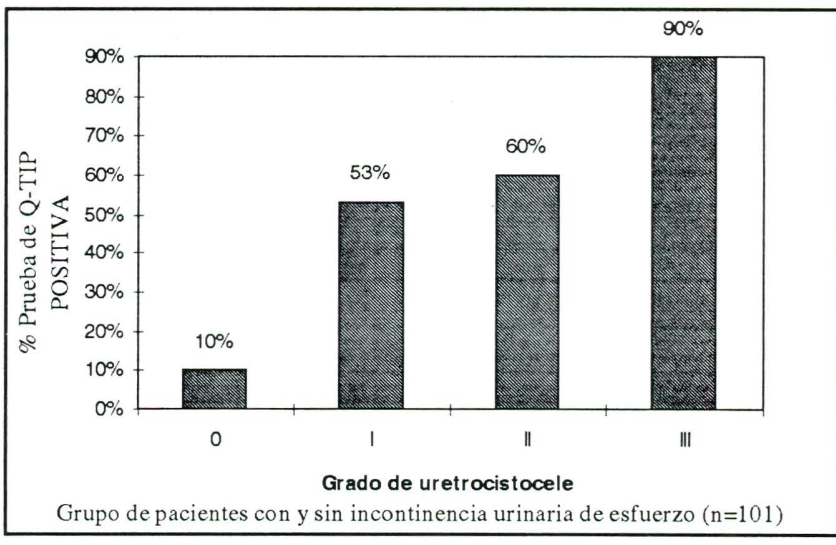

Se encontró que la prueba del Q-TIP es un método de muy baja sensibilidad y especificidad para el diagnóstico de incontinencia urinaria de esfuerzo, pero se encuentra estrechamente correlacionada en el grado de uretrocistocele de la paciente, resultados similares a los informados por otros autores $(3-4,11)$.

La prueba del Q-TIP por ser inespecífica, no importa información útil en el estudio de la paciente con incontinencia urinaria de esfuerzo, mayor a la obtenida con la historia clínica y el examen físico dirigido.

La utilidad del resultado de la prueba del Q-TIP para la elección de la técnica quirúrgica y como factor pronóstico de la efectividad de la cirugía,deberá ser establecida en futuros estudios.

\section{Conclusiones}

- La prueba del Q-TIP guarda estrecha correlación con el grado de uretrocistocele de la paciente.

- La prueba del Q-TIP no está relacionada con un diagnóstico urológico específico.

- La prueba del Q-TIP en la valoración de la mujer con incontinencia urinaria es inespecífica.

- El valor de la prueba del Q-TIP como método de predicción del tipo de corrección quirúrgica o pronóstico del resultado quirúrgico en la paciente con incontinencia urinaria de esfuerzo está por determinarse mediante futuros trabajos.

\section{BIBLIOGRAFIA}

1. Bergman A. Investigaciones e indicaciones del estudio urodinámico. Clin. Ginecol. Obstet. Temas actuales. 1989; 4: 779-786.

2. Crystle $\mathrm{C}$ et al. Q-TIP test in stress urinary incontinence. Obstet. Gymecol. 1971; 38: 313-315.

3. Walter M et al. Q-TIP Test: A study of continent and incontinent women. Obstet. Ginecol. 1987; 70: 208-211.

4. Montz F et al. Q-TIP test in female urinary incontinence. Obstet. Gynecol. 1986; 67: 258-260.

5. Bergman A et al. Comparison of three different surgical procedures for genuine stress incontinence: Prospective randomized study. Am. J. Obstet. Gynecol. 1989; 160: 1102-1106.

6. Summitt Ret al. Stress incontinence and low urethral closure pressure. J. Med. Reprod. Med. 1990; 35: 880-887.

7. Dávila G. Urinary incontinence in women. Postgrad Med. 1994; 96 : 103-110.
8. Bhatia $\mathrm{N}$ et al. Urodynamic effects of a vaginal pessary in women with stress urinary incontinence. Am. J. Obstet. Gynecol. 1983; 147: 876884.

9. Karram et al. The Q-TIP test: Standardization of the technique and its interpretation in women with urinary incontinence. Obstet. Gynecol. 1988; 71: 807-811.

10. Caputo $\mathrm{R}$ et al. The Q-TIP test and urethrovesical function mobility. Obstet. Gynecol. 1993; 82: 892-896.

11. Fantl $\mathbf{J}$ et al. Urethral axis and sphincteric function. Am. J. Obstet. Gynecol. 1986; 155: 554-558.

12. Mc Carthy T. Gynecologic Urology and Urodynamics. Ostergard. Williams-Wilkins, 1980. 